\section{Ciências sociais e fisioterapia: uma aproximação possível}

\section{The social sciences and physical therapy: a possible nexus}

\section{Patrícia Froes Meyer}

Docente da Universidade Federal do Rio Grande do Norte e Universidade Potiguar, Natal/RN Av. Gov. Sílvio Pedrosa, 200, ap. 1301 - Areia Preta 59014-100 Natal - RN - Brasil pffroes@terra.com.br

Íris do Céu Clara Costa

Professora de Pós-graduação em Ciências da Saúde da Universidade Federal do Rio Grande do Norte, Natal/RN Av. Senador Salgado Filho, 1787 - Lagoa Nova 59056-000 Natal - RN - Brasil irisdoceu@dod.ufrn.br

Vânia de Vasconcelos Gico

Professora e Pesquisadora dos Programas de Pós-Graduação em Ciências Sociais e em Ciências da Saúde da Universidade Federal do

Rio Grande do Norte, Natal/RN

Rua das Algarobas, 4 - Condomínio Residencial

Parque Itatiaia - Torre Bege, ap. 1501 Cidade Verde

59150-000 Parnamirim - RN - Brasil gico@digi.com.br
MEYER, P. F.; COSTA, I. do C. C.; GICO, V. de V.: Ciências sociais e fisioterapia: uma aproximação possível.

História, Ciências, Saúde - Manguinhos, Rio de Janeiro, v. 13 , n. 4, p. $877-90$, out.-dez. 2006.

O ensino das ciências sociais nos cursos da área de saúde tem sido objeto de reflexões e discussões. As várias dimensões do processo do adoecimento e a própria condição do doente, no aspecto não apenas biológico, mas psicossocial, escapam a uma simples análise biomédica, dada a sua complexidade. Esta pesquisa descritiva visou abordar as interfaces sociais, buscando a compreensão da saúde e da doença com base em uma concepção transdisciplinar, por meio da religação dos saberes através da disciplina sociologia da saúde no curso de Fisioterapia da Universidade Federal do Rio Grande do Norte. Concluímos que os docentes dessa disciplina, nos cursos da área da saúde, têm a responsabilidade de fazer o intercâmbio entre as ciências sociais e a prática do profissional da saúde, contextualizando o conhecimento à realidade da profissão na sociedade atual.

PALAVRAS-CHAVE: ciências sociais; fisioterapia; transdisciplinaridade; processo saúde-doença.

MEYER, P. F.; COSTA, I. do C. C.; GICO, V. de V.: The social sciences and physical therapy: a possible nexus.

História, Ciências, Saúde - Manguinhos, Rio de Janeiro, v. 13 , n. 4, p. 877-90, Oct.-Dec. 2006.

Recent scholarly reflections and discussions have been focused on the teaching of social sciences as part of courses in the health fields. The different dimensions involved in the process of taking ill and the state of being ill itself - not just from a biological but also from a psychosocial standpoint - transcend a simple biomedical analysis, given their complexity. The present descriptive research study, which analyzes data both quantitatively and qualitatively, endeavors to understand health and illness from an interdisciplinary view, i.e., where these fields of knowledge come together in the Sociology of Health class that is part of the Federal University of Rio Grande do Norte's Physical Therapy Course. The authors conclude that those who teach sociology as part of health field courses must assume the responsibility of linking the social sciences to the practice of healthcare workers, by placing knowledge within the context of the healthcare professional's reality in our society today.

KEYWORDS: social sciences; physical therapy; transdisciplinary; health-sickness process. 
$\mathrm{H}$ istoricamente, a formação escolar ensina a separar os objetos de seu contexto, as disciplinas umas das outras, dificultando a tentativa de relacioná-las. Essa separação e a fragmentação das disciplinas são incapazes de captar "o que está tecido junto", isto é, o complexo, segundo o sentido original do termo (Almeida \& Carvalho, 2002). Atualmente, percebe-se que as discussões nas escolas de formação biomédica têm sido favoráveis à religação dos saberes e a uma humanização dos atendimentos. A carência de um tratamento mais humanitário e social das pessoas que necessitam do profissional de saúde tem levado a repensar o ensino médico e de todas as outras áreas da saúde, também a fisioterapia, buscando a inclusão de novos olhares.

De acordo com Massey (2001), graduados em cursos da área de saúde devem ser capazes de trabalhar em equipe, com qualidade e compreensão. Um currículo transdisciplinar deve conter disciplinas e experiências práticas que busquem o conhecimento, habilidades e valores necessários ao trabalho em equipe de saúde. O currículo tradicional direciona seu foco para disciplinas específicas, sem a flexibilidade necessária a uma formação profissional direcionada à atenção comunitária. Esse tipo de currículo apresentase como um "currículo de doenças", segundo Finocchio \& Johnson (1995), que dificulta o crescimento e o desenvolvimento de uma formação transdisciplinar para o futuro fisioterapeuta.

A esse respeito, Capra (1992) afirma que a atenção centrada na doença conduz a uma noção de saúde relativa apenas aos aspectos orgânicos em suas especificidades, impedindo uma visão holística e uma intervenção que considere a totalidade do ser humano, em sua realidade social e considerando suas condições emocionais, sociais e culturais, que são parte da construção da doença. A importância dada à revolução científica e sua influência na área da saúde têm favorecido uma atenção centrada na doença e não no indivíduo, valorizando os aspectos curativos e deixando de lado os aspectos preventivos.

Infelizmente, a estrutura, a metodologia de ensino e a organização curricular ainda não estão baseadas nas demandas sociais e nas políticas públicas de saúde. A partir das análises situacionais quanto à implementação das diretrizes curriculares, da avaliação dos cursos de graduação e da capacitação docente, deliberou-se como referência de qualidade na graduação em fisioterapia no Brasil, a partir da Carta de Vitória (2004), que os currículos devem passar por uma adequação às necessidades regionais, e devem contemplar experiências práticas desde os períodos iniciais do curso, de forma a preparar o aluno para o estágio curricular, oportunizando a atuação fisioterapêutica em atenção primária, secundária e terciária, de forma equilibrada. Devem, também, estimular a inserção de docentes fisioterapeutas nas disciplinas ministrando conteúdos essenciais para 
o Curso de Graduação em Fisioterapia (Ciências Biológicas e da Saúde, Ciências Sociais e Humanas, Conhecimentos Biotecnológicos e Conhecimentos Fisioterapêuticos).

O ensino das ciências sociais nos cursos da área de saúde tem sido objeto de reflexões e discussões, seja nos âmbitos institucionais mais restritos, seja também nos fóruns de especialistas. Para Canesqui (2000), esse ensino traz aportes importantes à compreensão do significado dos problemas coletivos e individuais da saúde de grupos e segmentos sociais. As várias dimensões do processo do adoecimento e a própria condição do doente, no aspecto não apenas biológico, mas psicossocial, escapam a uma simples análise biomédica, dada a sua complexidade, tornando-se incapazes de se esgotarem nos olhares disciplinares.

Buscando uma nova abordagem dentro do curso de fisioterapia através da disciplina de Sociologia da Saúde, este trabalho pretendeu estimular os alunos a ampliarem a compreensão do processo saúde-doença durante as aulas dessa disciplina, das relações causae-efeito da vida em sociedade e de suas implicações na construção social, analisando, também, o papel da fisioterapia nesse processo. O discurso de uma nova abordagem do processo saúde-doença na formação do futuro profissional fisioterapeuta não é exatamente o que se pode chamar de novo, mas pode se chamar de 'inovadora' a idéia de se colocar em prática o ensino problematizado (Probleming Learning), baseado nos trabalhos de Richardson (1995) e Gross (1993) retomados por Ricieri (2004), numa disciplina do ciclo básico da formação acadêmica (sociologia) que irá favorecer as discussões das temáticas já citadas. Esta experiência visou proporcionar um conhecimento das interfaces sociais, buscando a compreensão da saúde e da doença a partir de uma concepção transdisciplinar, por meio da religação dos saberes. Estas reflexões, provavelmente, poderão desencadear uma maior humanização dos atendimentos prestados, uma melhor qualidade de vida para os pacientes, proporcionando assim uma atuação plena desses profissionais em relação à saúde.

O Problem-Base Learning (PBL), de origem inglesa, fundamentado na integração horizontal entre as ciências clínicas e básicas, trabalha a interdisciplinaridade de conteúdos dentro de um mesmo período ou série, e a transdisciplinaridade, através da integração de conteúdos de um período ou série a outro, desenvolvendo no aluno a capacidade de transmigrar o conhecimento adquirido para além dos limites da disciplina, bem como ultrapassar a forma como tais conteúdos foram organizados didaticamente para serem transmitidos. Assim, essa modalidade de ensino não é exatamente centrada no aluno, embora já comece a apresentar os primeiros movimentos para se libertar do modelo tradicionalista centrado no professor (Ricieri, 2004). 
O fato de as ciências sociais se inserirem geralmente ao lado das disciplinas básicas conduz algumas experiências brasileiras a organizarem seus conteúdos com base na seleção de assuntos ou de grupos de patologias, em torno dos quais são discutidas as várias dimensões do processo do adoecimento. Isto é, problematizam-se as concepções da doença dadas pelo modelo biomédico e a própria condição do doente, recorrendo aos estudos sociológicos, históricos e antropológicos. Essa experiência requer grande flexibilidade curricular e dinâmica no ensino, mas quando acontece, é capaz de permitir que as ciências sociais ofereçam reflexões que lhes são próprias sobre certos objetos que escapam às análises das ciências biomédicas.

Na prática, os currículos do Curso de Fisioterapia estão distantes de uma preocupação com essas questões, pois ainda não há nada de concreto em vigor nas universidades. De acordo com Carvalho (1999), considera-se uma falha na formação do fisioterapeuta a falta de direcionamento das disciplinas para as deficiências da área, para a preocupação com a reintegração social do paciente, para a preocupação com o mercado de trabalho. Faltam também conteúdos mais preventivos que curativos, questões de funcionalidade e restrições que devem ser orientadas ao aluno, além da viabilização de ações na formação dos fisioterapeutas que lhes permitam um envolvimento com políticas institucionais e governamentais, principalmente em relação a pessoas deficientes. É necessária também a busca de um envolvimento mais amplo com a realidade das pessoas portadoras das patologias amparadas pelo fisioterapeuta. O trabalho do profissional fisioterapeuta deve voltar-se, portanto, para a promoção da saúde, de forma global, especialmente se ele pretende trabalhar numa equipe transdisciplinar.

\section{Metodologia}

Trata-se de uma pesquisa descritiva, com análise de dados de forma quantitativa e qualitativa, baseada nos autores Bauer \& Gaskel (2002), que confirmam essas idéias afirmando não haver quantificação sem qualificação, ou seja, não haver análise estatística sem interpretação. É necessária uma visão holística do processo de pesquisa para que ele possa incluir a definição e a revisão de um problema, sua teorização, a coleta de dados e a apresentação dos resultados. A análise metodológica também se baseou nos estudos de Minayo (2000), que sugerem a necessidade de uma pesquisa qualitativa ancorada nos estudos que privilegiam a religação na construção do conhecimento, representado neste trabalho pelos aportes transdisciplinares e pela busca da humanização da saúde.

Como instrumento para coleta de dados foi utilizado um questionário de perguntas abertas ou livres, criado em consonância 
com os objetivos desta pesquisa, dando liberdade ao aluno para expressar livremente suas opiniões. Quando analisadas com cuidado, as respostas abertas podem ser transformadas em variáveis e unidas ao conjunto dos dados quantitativos. A categorização das respostas, de acordo com Bauer \& Gaskel (2002), foi realizada através da seleção das palavras-chave e das 'falas' mais expressivas. Uma vez homogeneizados a partir da similaridade semântica, os dados foram examinados com respeito às palavras relevantes, através do emprego de uma lista de palavras ordenadas por sua freqüência.

O questionário foi submetido ao processo de validação de acordo com a orientação de Lisboa (2000), passando pelas estratégias de painel de experts e de validade de face. Na validade de face foram consultados cinco profissionais que atuam em diversas áreas da fisioterapia, selecionados aleatoriamente, para responder a um protocolo de validade de face. As perguntas abordaram possíveis dificuldades na leitura do questionário de questões abertas, o nível de compreensão das questões para os estudantes de fisioterapia, a presença de erros de tipografia, o tamanho da letra e a extensão do questionário, bem como o tempo de aplicação e a avaliação geral.

Na validade de conteúdo (painel de experts) foram consultados três profissionais especialistas em ciências humanas (uma psicóloga, um cientista social e um filósofo), selecionados de forma aleatória, fazendo seu julgamento 'técnico' a respeito da inclusão ou exclusão de questões no questionário.

Após o processo de validação e a realização das modificações sugeridas foi aplicado um pré-teste a 27 alunos do curso de Fisioterapia da Universidade Potiguar em Natal (RN), acadêmicos de diversos períodos, buscando verificar se ainda existiam falhas no instrumento, além de avaliar sua operatividade e linguagem.

As percepções e observações obtidas durante as aulas foram redigidas nas notas de campo. Estas, de acordo com Triviños (1987), são descrições, comentários, reflexões, idéias e questionamentos que surgem no decorrer da pesquisa e que vão sendo organizados e desenvolvidos durante o processo investigatório. As notas de campo foram redigidas no diário de campo pelo pesquisador, logo após as discussões, comentários verbais e acontecimentos considerados relevantes.

Os indivíduos investigados são alunos matriculados na Disciplina de Sociologia da Saúde no segundo período do curso de Fisioterapia da Universidade Federal do Rio Grande do Norte, em Natal, somando 21 alunos de faixa etária entre 18 e 28 anos, submetidos à pesquisa no período de março a julho de 2003. 


\section{Resultados e discussões}

O Quadro 1 mostra a programação das aulas de Sociologia da Saúde, organizada em momentos:

Quadro I: Programação das temáticas e metodologia utilizada na disciplina de Sociologia da Saúde no primeiro semestre de 2003, Natal/RN

\begin{tabular}{|c|c|c|}
\hline Período & Temáticas & Metodologia \\
\hline I Momento & Perfil do grupo & $\begin{array}{l}\text { Questionários e } \\
\text { dinâmicas de grupo } \\
\text { Debates, leitura de } \\
\text { imagens, textos, } \\
\text { filmes, painéis }\end{array}$ \\
\hline II Momento & $\begin{array}{l}\text { Homem e Sociedade } \\
\text { Divisão de trabalho e } \\
\text { classes sociais }\end{array}$ & \\
\hline III Momento & $\begin{array}{l}\text { A saúde como } \\
\text { processo social }\end{array}$ & $\begin{array}{l}\text { Filme, textos, } \\
\text { discussões, } \\
\text { dinâmicas de grupo }\end{array}$ \\
\hline IV Momento & $\begin{array}{l}\text { A contribuição da ciências } \\
\text { sociais no campo da saúde } \\
\text { A fragmentação do } \\
\text { conhecimento }\end{array}$ & $\begin{array}{l}\text { Filme, trabalho de } \\
\text { campo, seminários }\end{array}$ \\
\hline V Momento & $\begin{array}{l}\text { Humanização da saúde } \\
\text { Reavaliação }\end{array}$ & $\begin{array}{l}\text { Relatório do } \\
\text { trabalho de campo, } \\
\text { questionários e } \\
\text { auto-avaliação }\end{array}$ \\
\hline
\end{tabular}

Fonte: Plano de ensino da disciplina de Sociologia da Saúde do curso de Fisioterapia 2003.1, Universidade Federal do Rio Grande do Norte, Natal, RN.

\section{a) Primeiro momento: caracterização do grupo e expectativas em relação à disciplina; questionamento sobre sua importância}

Quanto à escolha do curso, 60 por cento dos alunos apontaram a Fisioterapia como primeira opção no vestibular, pois realmente queriam essa profissão, enquanto 40 por cento vinham de vestibulares frustrados em Medicina (29\%) e Odontologia (11\%). Apesar dessa característica, todos os pesquisados se mostravam satisfeitos com o curso. Em relação à expectativa em torno da disciplina, havia uma opinião influenciada pelas turmas anteriores de que se tratava de uma disciplina sem importância e, principalmente, sem nenhuma ligação com a fisioterapia. As respostas mais encontradas nos questionários eram: "não sei para que serve, espero descobrir ao 
final da disciplina", "deve ter alguma finalidade, pois do contrário não seria disciplina obrigatória", "ouvi falar que é muito cansativa".

Solicitou-se aos alunos que indicassem as três disciplinas mais importantes e as três menos importantes no Curso de Fisioterapia. $\mathrm{O}$ resultado pode ser visto nas Tabelas 1 e 2:

Tabela I: Distribuição de freqüência das disciplinas mais importantes para o curso de Fisioterapia segundo os estudantes, na aplicação prévia do questionário, Natal/RN, 2003

\begin{tabular}{lc}
\hline Disciplina & Freqüência \\
\hline Cinesiologia & 12 \\
Anatomia & 09 \\
Fisiologia & 06 \\
Cinesioterapia & 05 \\
Fisiopatologia & 04 \\
Patologia & 02 \\
Biologia celular & 02 \\
Fisiopatologia em ortopedia e traumatologia & 02 \\
Hidroterapia e mecanoterapia & 02 \\
Imunologia & 02 \\
Todas as disciplinas são importantes & 07 \\
\hline
\end{tabular}

Fonte: pesquisa de campo.

Tabela 2: Distribuição de freqüência das disciplinas menos importantes para o curso de Fisioterapia segundo os estudantes, na aplicação prévia do questionário, Natal/RN, 2003

\begin{tabular}{lc}
\hline Disciplina & Freqüência \\
\hline Filosofia & 03 \\
Antropologia & 03 \\
Psicologia & 03 \\
Bioestatística & 02 \\
Sociologia & 02 \\
Metodologia da pesquisa científica & 02 \\
Não se lembra ou não sabe & 02 \\
Não há disciplina sem importância & 06 \\
\hline
\end{tabular}

Fonte: pesquisa de campo.

Nas Tabelas 1 e 2 excluíram-se as disciplinas que receberam apenas um voto, pois seu grande número dificultaria a visualização dos resultados. Os dados demonstram a predominância das disciplinas 'técnicas' como as mais importantes e das disciplinas classificadas como 'humanísticas' como as menos importantes, confirmando as citações de Rebelatto \& Botomé (1999). Embora haja um ciclo de matérias de formação geral (incluindo disciplinas de ciên-cias sociais e humanas) que abrangem os estudos do homem, de suas relações sociais, dos processos saúde-doença, parece ocorrer grande resistência por parte 
dos alunos da área de saúde a essas disciplinas. A preocupação excessiva com as técnicas impede que o fisioterapeuta enxergue o ser humano que está diante dele.

Examinando as disciplinas que compõem o currículo de formação do fisioterapeuta, encontra-se uma lacuna que pode interferir na sua atuação. Há uma significativa ausência de disciplinas voltadas para a formação científica, o que leva ao risco de se formarem "consumidores de manuais" e pessoas que lêem no máximo a literatura técnica que freqüentemente acompanha os equipamentos. Tudo isso favorece o distanciamento do aluno em relação às questões mais complexas, pois de maneira geral, os fisioterapeutas são desencorajados das abordagens mais amplas e diversificadas. Devem-se levar em conta as limitações dos estudos generalizantes, embora fique evidente o desestímulo ao exercício da reflexão do todo, em que os fenômenos particulares se desenvolvem (Medina, 1998). Além disso, a preocupação quase exclusiva com a patologia, com a terapia e com as técnicas fisioterápicas constitui, de certa forma, uma clara negação e ignorância das múltiplas contribuições para se definir a atuação em fisioterapia. Provavelmente, énesse momento do curso que o aluno deve ser estimulado a pensar diferente, pois se ele não se motivar através da reflexão durante a sua formação, futuramente poderá tornar-se um profissional limitado perante a sociedade.

b) Segundo, terceiro e quarto momentos: homem e sociedade; divisão de trabalho e classes sociais; a saúde como processo social; a contribuição das ciências sociais no campo da saúde; a fragmentação do conhecimento e a humanização da saúde

Tendo em vista a falta de familiaridade com as ciências sociais, havia certa dificuldade quanto à assimilação do conteúdo e ao entendimento dos textos, tendo surgido a necessidade de mais tempo para efetuar as leituras. Inicialmente, havia certa perplexidade em relação às temáticas, gerando comentários como "Será que eu preciso saber tudo isto para ser fisioterapeuta?". Para facilitar a compreensão dos textos e dos assuntos discutidos recorremos aos recursos didáticos: filmes, dinâmicas de grupo, trabalhos em pequenos grupos, discussões e reflexões, por exemplo.

Foi indispensável a relação entre a teoria estudada na disciplina e a prática profissional da fisioterapia. A relação desses conteúdos com as habilidades específicas do fisioterapeuta serviu também como instrumento didático-pedagógico, fortalecendo a convicção de que é preciso, segundo Paim (1993), "pensar para agir e agir para pensar melhor".

Os diálogos professor/aluno e aluno/aluno enfatizaram que os profissionais da saúde, hoje, apresentam como características a perda 
da humanização de seus atendimentos, o excesso tecnológico da assistência e a superespecialização das áreas, que resultam mais em uma atuação ligada à realização de tratamentos do que a cuidados propriamente ditos. A compreensão dos alunos sobre o processo saúde-doença gerou as seguintes discussões sobre o tema: "a troca de saberes em diversas áreas facilita a compreensão do processo saúde-doença"; "a saúde está intimamente relacionada à desigualdade"; " a população deve participar dos processos saúde-doença, pois se trata de sua própria saúde e vida"; "o processo saúde-doença está também na dependência do processo dominação-subordinação"; "o indivíduo doente é fruto de sua vida em sociedade".

Após essa reflexão, realizou-se discussão do tema relacionando à fisioterapia: como o fisioterapeuta entende a saúde e a doença? Pediu-se que cada grupo associasse uma palavra como resposta a essa questão, e a união dos grupos e das palavras transformou as palavras em uma frase que respondesse à questão: "O fisioterapeuta deve olhar para toda a dimensão da saúde que ultrapassa o contexto de doenças e suas causas, deve possuir uma visão global do ser humano, com um perfil que vai além da reabilitação".

A temática do quarto momento associou temas como humanização, transdisciplinaridade e fragmentação do homem na saúde, e, após as discussões a respeito desses assuntos, obtivemos as seguintes impressões:

"Conhecendo nosso verdadeiro papel, nosso trato com os pacientes passa a ser com os olhos da sociedade";

“Percebemos que não adianta apenas um tratamento clínico, é necessário um tratamento humanizado";

“Não adianta o fisioterapeuta cuidar dos ossos ou dos músculos do paciente, ele tem que cuidar do paciente";

“Quando o fisioterapeuta se preocupa com o bem-estar não só físico, mas psíquico e social do paciente, ele está demonstrando a importância do trabalho em equipe";

"Ser um profissional transdisciplinar significa ousar, não querer apenar curar o doente, mas trazê-lo novamente à vida em sociedade. É tornar-se capaz de enxergar novos horizontes, muitos deles esquecidos por outros profissionais".

Os conceitos de humanização, transdisciplinaridade e fragmentação foram facilmente entendidos e amplamente discutidos. Também se enfatizou a importância do conhecimento científico para o profissional de saúde e o quanto é difícil romper com as regras da formação positivista e tradicional da medicina para atuar de uma forma mais complexa.

As respostas tornaram possível verificar, em vários momentos das aulas, que os alunos apresentavam agora uma compreensão 
mais ampla do processo saúde-doença, considerando o paciente em toda sua complexidade. Para Morin (2002), atuar com humanidade permite reconhecer o humano no outro como ser complexo, tornar-se apto a situar-se no mundo em sua própria terra, sua história, sua sociedade. Não basta enunciar as necessidades de se contextualizar o ensino para esses acadêmicos e de religar seus saberes, é preciso colocar tudo isso em prática, o que provavelmente será possível através da disciplina Sociologia da Saúde, dentro desta visão transdisciplinar.

\section{c) Quinto momento: reavaliação}

O mesmo questionário foi reaplicado no encerramento do período letivo, para que os alunos respondessem às mesmas questões abordadas previamente, quando estavam iniciando a disciplina e ainda não conheciam os conteúdos a serem abordados. Após quinze encontros, as discussões provavelmente provocaram mudanças na maneira pela qual esses alunos olhavam a fisioterapia. Os temas abordados foram, entre outros: indivíduo, cultura e sociedade; a natureza social do homem; a construção histórico-social da realidade; a divisão de trabalho e classes sociais na sociedade capitalista; a divisão social do trabalho na fisioterapia; a saúde como processo social; a fragmentação do homem; transdisciplinaridade e humanização da saúde; a contribuição das ciências sociais no campo da saúde; as condições de saúde no Brasil e as políticas de saúde; o papel do fisioterapeuta na sociedade e a história da Fisioterapia no Rio Grande do Norte.

Solicitou-se, como no primeiro momento, a opinião dos pesquisados a respeito das disciplinas consideradas mais e menos importantes para o curso de fisioterapia. Os resultados constam nas Tabelas 3 e 4:

Tabela 3: Distribuição de freqüência das disciplinas mais importantes para o curso de Fisioterapia segundo os estudantes, na aplicação posterior do questionário, Natal/RN, 2003

\begin{tabular}{lc}
\hline Disciplina & Freqüência \\
\hline Sociologia & 03 \\
Psicologia & 03 \\
Anatomia & 02 \\
Ortopedia e traumatologia & 02 \\
Fisiologia & 02 \\
Fisioterapia respiratória & 02 \\
Fisioterapia aplicada a cardiologia e angiologia & 02 \\
Não sabe & 02 \\
Todas as disciplinas são importantes, tanto & 15 \\
humanísticas quanto da saúde & \\
\hline
\end{tabular}

Fonte: pesquisa de campo. 
Tabela 4: Distribuição de frequiência das disciplinas menos importantes para o curso de Fisioterapia segundo os estudantes, na aplicação posterior do questionário, Natal/RN, 2003

\begin{tabular}{lc}
\hline Disciplina & Freqüência \\
\hline Histologia & 02 \\
Psicologia & 02 \\
Antropologia & 02 \\
História da Fisioterapia & 02 \\
Administração & 02 \\
Não há disciplina menos importante & 16 \\
\hline
\end{tabular}

Fonte: pesquisa de campo.

As respostas dos alunos ao questionário mesclam comentários reconhecendo a relevância das disciplinas em geral, colocando no mesmo nível as humanísticas e as técnicas. Em relação às respostas, a questão que aborda a importância da disciplina Sociologia para o Curso de Fisioterapia recebeu a confirmação unânime dessa importância. As justificativas mais freqüentes estão reunidas na Tabela 5:

Tabela 5: Importância da disciplina de Sociologia no curso de Fisioterapia segundo a opinião dos alunos da UFRN, Natal/RN, 2003

\begin{tabular}{lc}
\hline Importância da disciplina de Sociologia & Freqüência \\
\hline $\begin{array}{l}\text { Conhecer a realidade social do paciente e poder } \\
\text { analisa-lo como um “todo” para propor um tratamento eficaz }\end{array}$ & 7 \\
$\begin{array}{l}\text { Proporcionar um conhecimento que integra a Fisioterapia e a } \\
\text { sociedade, pois o profissional precisa conhecer a realidade de } \\
\text { cada um e suas relações. }\end{array}$ & 6 \\
Ter uma visão mais humanizada da saúde & 5 \\
Entender melhor o trabalho em equipe de saúde & 3 \\
$\begin{array}{l}\text { Aprendemos a valorizar também outras disciplinas } \\
\text { como a antropologia, psicologia, ética etc... } \\
\text { Conhecer a realidade profissional do Fisioterapeuta }\end{array}$ & 3 \\
\hline
\end{tabular}

Fonte: pesquisa de campo.

A maioria das expectativas manifestadas no primeiro dia de aula foi contemplada nas respostas dos alunos. Houve também uma reivindicação por aumento de carga horária dessa disciplina e a solicitação da participação constante de um fisioterapeuta na construção e durante o decorrer da disciplina. Desta forma, segundo os alunos, torna-se possível estabelecer a ponte entre a teoria e a prática profissional, o que despertou muito interesse para a participação nas aulas e um esclarecimento maior do conteúdo.

Para os pesquisadores Strohschein et al. (2002), há uma dicotomia entre formação profissional e atuação técnica do fisioterapeuta, além de os autores serem contrários a uma filosofia de educação isolada. A construção da educação requer transdisciplinaridade e abordagem holística direcionadas às necessidades dos estudantes 
enquanto cidadãos. Baugniet et al. (2000) afirmam que os estudantes geralmente percebem uma inconsistência entre teoria e prática. A proposta de um currículo transdisciplinar, segundo os autores, estimula o aprendizado fora da universidade, facilitando a ponte entre $o$ conhecimento teórico e prático e estimulando a formação de futuros profissionais mais reflexivos e críticos, com importante impacto na cultura e na sociedade. Teoricamente, o estu-dante que se engajar nesse processo terá de iniciar o aprendizado de habilidades como a reflexão em equipe.

\section{Conclusões}

Diante do que foi aqui exposto, tanto o conhecimento da fisioterapia quanto a sua atividade prática na universidade devem estar situados dentro da interação de atividade/contexto/cultura e devem ser desenvolvidos envolvendo a complexidade social. Quanto mais o conhecimento for apenas 'reproduzido' e 'transmitido', em vez de também ser produzido, levando em consideração a realidade circundante, mais longe estarão os futuros profissionais de obter resoluções para os problemas da população que os procura. Dessa forma, a concepção de universidade como uma organização capaz de produzir conhecimento e como local de debates sobre as soluções alternativas para os problemas do país, perde terreno para essa concepção acentuadamente reprodutiva e transmissora de informações práticas, em geral, técnicas.

As medidas que podem auxiliar na transformação do ensino em fisioterapia partem de uma nova estruturação de conteúdos, um redimensionamento das áreas em redes conectadas e interdependentes, mostrando dentro do curso um verdadeiro exercício de transdisciplinaridade. Para a educação do futuro, todavia, será necessária uma maior amplitude na condução do trabalho acadêmico, ao considerar suas incertezas e limitações e tendo em vista o sujeito como participante ativo de todo o processo. Baseando-se nos pensamentos de Morin (2001a), de que todo conhecimento comporta o risco do erro e da ilusão, não se pode subestimar o que ocorre em todo processo de mudança - dificuldades e riscos incertos.

Reconhecemos ainda que a contribuição das Ciências Sociais não se dá apenas na reflexão do processo saúde-doença, embora esse tema tenha relevância e ocupe muitas discussões. Sugere-se levar o estudante a um maior entendimento da historicidade da fisioterapia, da organização da prática fisioterapêutica, do trabalho e da profissão, como uma das questões de maior importância e que requerem uma reforma do pensamento, como pensa Morin (2001b). Só assim incidirão transformações sobre as intervenções políticas, sociais e educacionais, ou seja, os múltiplos processos sociais que são de interesse da sociedade como um todo e das questões profissionais 
do fisioterapeuta. Não podemos esquecer que as questões de saúde em nossa sociedade também são sociais e políticas e mobilizam problemas culturais, religiosos e envolvem outros campos da saúde. Essa sugestão não descarta o ensino da Sociologia associado a programas de atenção comunitária, de ordem primária, sem desfigurar sua especificidade, pois ele trará aportes importantes à compreensão do significado dos problemas coletivos e individuais de saúde de grupos e segmentos sociais.

Concluímos, também, que os docentes da disciplina Sociologia dos cursos da área da saúde, entre eles fisioterapia, têm a responsabilidade de facilitar a reflexão com abordagens problematizadoras, fazendo o intercâmbio entre as ciências sociais e a prática profissional, contextualizando o conhecimento à realidade da profissão na sociedade atual. Empenhando-se dessa forma, a disciplina Sociologia irá contribuir para o desenvolvimento da capacidade de resolução de problemas pelo futuro profissional que provavelmente irá encontrar um mercado de trabalho exigente e competitivo, repleto de recursos tecnológicos e submerso em um mar de informações. Além disso, a visão transdisciplinar esperada na formação do fisioterapeuta poderá preparar esse aluno para a cidadania, enfatizando a preocupação com o respeito aos colegas e pacientes e com a qualidade de vida, desenvolvendo a habilidade do trabalho em equipe, dentro das comunidades, o pensamento crítico, a capacidade de solucionar problemas dentro da equipe e da comunidade e o interesse em pesquisas com aplicação dentro de uma equipe de saúde. Desta forma, a imagem que o aluno de fisioterapia tem das ciências sociais e humanas poderá modificar-se para melhor.

\section{REFERÊNCIAS BIBLIOGRÁFICAS}

Almeida, Maria da Conceição; Carvalho, Edgard de Assis (org.) 2002

Bauer, Martin W.; Gaskel, George 2002

Baugniet, Jessica; Boon, Heather; Ostbye, Truls 2000

Canesqui, Ana Maria 2000

Capra, Fritjof 1992

Carta de Vitória 2004
Educação e complexidade: os sete saberes e outros ensaios - Edgar Morin. São Paulo: Cortez. 102 p.

Pesquisa qualitativa com texto, imagem e som. Manual Prático. Petrópolis: Vozes.

Complementary/Alternative Medicine: Comparing the views of medical students with students in other health care professions. Medical Student Education, v. 32, n. 3, March, p. 178-84.

Ciências sociais e saúde para o ensino médico.

São Paulo: Hucitec. 287 p.

O ponto de mutação.

São Paulo: Cultrix.

(Resoluções do Congresso Brasileiro de Ensino em Fisioterapia).

Disponível em www.abenfisio.com.br, acesso em agosto de 2004. 
Carvalho, Cecília

Barros

1999

Finocchio, L; Johnson, Carl 1995

Gross, V. 1993

Lisboa, Fábio 2000

Massey, Carole 2001

Medina, João Paulo 1998

Minayo, Maria Cecília de Souza 2000

Morin, Edgar 2002

Morin, Edgar 2001a

Morin, Edgar 2001b

Paim, J. S. 1993

Rebelatto, José Rubens; Botomé, Sílvio 1999

Ricieri, Denise de Vinha 2004

Richardson, B. 1995

Sthroschein, Jennifer; Hagler, Paul; May, Laura 2002

Triviños, A. N. S. 1987
Análise do preparo do fisioterapeuta para atuar e intervir na área de reabilitação profissional. Salusvita, Bauru (SP), v. 18, n. 2, p. 7-23.

A Core Curriculum in Allied Health Education: Current issues and future expectations. Journal of Allied Health, v. 24, p. 203-19.

Introducing physiotherapy students to the idea of reflective practice. Medical Teacher, v. 15, n. 4, p. 293-307.

Survey of Best Physical Education Pratices for Children with Autistic Behaviours. Tese de doutoramento. Escola de Graduação da Ohio State University (EUA). Columbus.

A Transdisciplinary Model for Curricula Revision. Nursing and Health Care Perspectives, v. 22, n. 2, March, p. 85-98.

O brasileiro e seu corpo.

5. ed. Campinas: Papirus.

O desafio do conhecimento: pesquisa qualitativa em saúde.

7. ed. São Paulo: Hucitec.

A religação dos saberes: o desafio do século XXI.

2. ed. Rio de Janeiro: Bertrand Brasil. 583 p.

Os sete saberes necessários à educação do futuro.

3. ed. São Paulo: Cortez. 118 p.

A cabeça bem feita: repensar a reforma, reformar o pensamento.

3. ed. Rio de Janeiro: Bertrand Brasil. 98 p.

Marco de referência para um programa de educação continuada em Saúde Coletiva. Revista Brasileira de Educação Médica, v. 17, p. 7-13.

Fisioterapia no Brasil.

2. ed. São Paulo: Manole.

A Abordagem Problematizadora no Ensino da Fisioterapia.

Fisiobrasil, v. 66, jul./ago.

A Study of the thoughts and reflections of physiotherapists during the first year of graduation. Abstracts of Papers of 12th International Congress of the World Confederation for Physical Therapy, Washington, USA.

Assessing the need for change in clinical education practices. Physical Therapy, v. 82, n. 2, February. p. 160-72.

Introdução à pesquisa em ciências sociais: a pesquisa qualitativa em educação. São Paulo: Atlas.

Recebido para publicação em novembro de 2004.

Aprovado para publicação em junho de 2005. 